\title{
Current attitude of rheumatology practitioners on transition and transfer from pediatric to adult health care
}

\author{
Deborah Hilderson ${ }^{1,2^{*}}$, Philip Moons ${ }^{1}$, Rene Westhovens ${ }^{3}$, Carine Wouters ${ }^{4}$ \\ From 18th Pediatric Rheumatology European Society (PReS) Congress \\ Bruges, Belgium. 14-18 September 2011
}

\section{Background}

When patients with a rheumatic disorder reach adulthood, transfer to adult-focused care is advocated (1). A successful continuation of rheumatology follow-up beyond childhood depends on how clinicians think about transfer and transition (2).

\section{Aim}

To explore the attitudes of rheumatology practitioners on transfer and transition from pediatric to adult health care of adolescents with a rheumatic disorder.

\section{Method}

A survey (validated QUARTT, QUestionnaire about Attitudes of Rheumatology practitioners towards Transfer and Transition) among rheumatology practitioners attending the Pediatric Rheumatology European Society congress in 2010. Overall, 138 individuals participated, of which 59 were medical doctors (MDs) with paediatric and rheumatology background; 30 MDs with pediatric background; and 21 MDs with rheumatology background.

\section{Results}

Participants judged that, when patients with active rheumatic disorder reach adulthood, they should receive medical follow-up from an adult rheumatologist (87\%). Only $19 \%$ thought that patients should stay under surveillance of a paediatric rheumatologist. Several initiating factors for transfer were marked as important: readiness of the patient according to the caregiver (62\%), age (61\%) and psychosocial maturity (49\%). A transfer meeting with the patient $(76 \%)$, a referral letter $(73 \%)$, or a medical transfer file $(64 \%)$ were most preferred transfer communication tools with adult healthcare. Joint outpatient clinic, phone calls, and transfer meetings without the patient were considered to be less useful. The pediatric (94\%) or adult rheumatologist $(83 \%)$, parents $(81 \%)$ and the nurse specialist $(74 \%)$ were stated as most important active participants in the transition process.

\section{Conclusion}

This study emphasized the importance of transfer to specialized rheumatology care of adolescents with an active rheumatic disease and highlighted transfer initiators and transfer communication tools.

\begin{abstract}
Author details
${ }^{1}$ Center for Health Services and Nursing Research, Katholieke Universiteit Leuven, Leuven, Belgium. 'Division of Pediatrics, University Hospitals Leuven, Leuven, Belgium. ${ }^{3}$ Rheumatology, Department of Musculoskeletal Sciences, University Hospitals Leuven, Leuven, Belgium. ${ }^{4}$ Pediatric Rheumatology, University Hospitals Leuven, Leuven, Belgium.
\end{abstract}

Published: 14 September 2011

doi:10.1186/1546-0096-9-S1-P274

Cite this article as: Hilderson et al:: Current attitude of rheumatology practitioners on transition and transfer from pediatric to adult health care. Pediatric Rheumatology 2011 9(Suppl 1):P274.

\footnotetext{
* Correspondence: deborah.hilderson@med.kuleuven.be

${ }^{1}$ Center for Health Services and Nursing Research, Katholieke Universiteit Leuven, Leuven, Belgium

Full list of author information is available at the end of the article
} 